\title{
Knot Group Epimorphisms
}

\section{DANIEL S. SILVER and WILBUR WHITTEN}

\begin{abstract}
Let $G$ be a finitely generated group, and let $\lambda \in G$. If there exists a knot $k$ such that $\pi k=\pi_{1}\left(S^{3} \backslash k\right)$ can be mapped onto $G$ sending the longitude to $\lambda$, then there exists infinitely many distinct prime knots with the property. Consequently, if $\pi k$ is the group of any knot (possibly composite), then there exists an infinite number of prime knots $k_{1}, k_{2}, \cdots$ and epimorphisms $\cdots \rightarrow \pi k_{2} \rightarrow \pi k_{1} \rightarrow \pi k$ each perserving peripheral structures. Properties of a related partial order on knots are discussed.
\end{abstract}

1. Introduction. Suppose that $\phi: G_{1} \rightarrow G_{2}$ is an epimorphism of knot groups preserving peripheral structure (see $\S 2$ ). We are motivated by the following questions.

Question 1.1. If $G_{1}$ is the group of a prime knot, can $G_{2}$ be other than $G_{1}$ or $\mathbf{Z}$ ?

Question 1.2. If $G_{2}$ can be something else, can it be the group of a composite knot?

Since the group of a composite knot is an amalgamated product of the groups of the factor knots, one might expect the answer to Question 1.1 to be no. Surprisingly, the answer to both questions is yes, as we will see in $\S 2$.

These considerations suggest a natural partial ordering on knots: $k_{1} \geq k_{2}$ if the group of $k_{1}$ maps onto the group of $k_{2}$ preserving peripheral structure. We study the relation in $\S 3$.

2. Main result. As usual a knot is the image of a smooth embedding of a circle in $S^{3}$. Two knots are equivalent if they have the same knot type, that is, there exists an autohomeomorphism of $S^{3}$ taking one knot to the other.

Let $k$ be a knot in $S^{3}$. We denote its group $\pi_{1}\left(S^{3} \backslash \operatorname{int} V, *\right)$ by $\pi k$. Here $V \cong k \times D^{2}$ is a tubular neighborhood of $k$, and the basepoint $*$ is chosen on the boundary $\partial V \cong k \times S^{1}$. The element $m$ represented by a simple closed curve in $\partial V$ that is contractible in $V$ is called a meridian; the element $l$ represented by a simple closed curve in $\partial V$ that is nullhomologous in $S^{3} \backslash \operatorname{int} V$ is called a longitude. A well-known algorithm enables one to express $l$ in terms of Wirtinger generators corresponding to a diagram for $k$. Details can be found on page 37 of [BZ85].

The inclusion map $\partial V \hookrightarrow S^{3} \backslash \operatorname{int} V$ induces an injection of fundamental groups. Its image is the subgroup $\langle m, l\rangle$ generated by $m$ and $l$.

First author is partially supported by NSF grant DMS-9704399.

2000 Mathematics Subject Classification. Primary 57M25; secondary 37B10. 
Let $k_{i}, i=1,2$, be knots with meridian-longitude pairs $m_{i}, l_{i}$. A homomorphism $\pi k_{1} \rightarrow \pi k_{2}$ preserves peripheral structure if the image of $\left\langle m_{1}, l_{1}\right\rangle$ is conjugate to a subgroup of $\left\langle m_{2}, l_{2}\right\rangle$.

Definition 2.1. (i) $k_{1}$ covers $k_{2}$ (or $k_{2}$ supports $k_{1}$ ) if there is an epimorphism $\pi k_{1} \rightarrow \pi k_{2}$;

(ii) If $G$ is a finitely generated group normally generated by an element $\mu$, and if $\lambda \in G$, then a knot $k$ covers $(G, \mu, \lambda)$ (or briefly $k$ covers $G$ ) if there exists an epimorphism $\phi:(\pi k, m, l) \rightarrow(G, \mu, \lambda)$, where $(m, l)$ is a meridian-longitude pair.

If $k$ covers $G$ for a given $\lambda \in G$, then we say (after Johnson and Livingston [JL89]) that $k$ realizes $\lambda$. For a given group $G$ as above and $\lambda \in G$, [JL89] provides necessary and sufficient conditions for the existence of a knot $k$ that covers $(G, \mu, \lambda)$. We will show that $k$ can always be chosen to be a prime knot.

Theorem 2.2. Let $G$ be a finitely generated group that is normally generated by a single element $\mu$, and let $\lambda \in G$. If there exists a knot $k$ that realizes $\lambda$, then there exists an infinite number of distinct prime knots that realize $\lambda$.

Proof. As the theorem easily follows when $k$ is trivial, we assume that $k$ is knotted. By Proposition 2.5 of [EKT03], we can regard $k$ as the numerator closure $T^{N}$ of a prime tangle $T$. (See [EKT03], where the authors provide general terminology and prove an even stronger condition). Set $C=(1 / 2)^{N}$, using Conway notation (Figure 1).

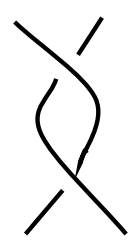

Figure 1: The tangle 1/2

We use a construction of [EKT03] to form a 2-component link $L=T^{N} \cup(1 / 2)^{N}=k \cup C$ (Figure 2). The components $k$ and $C$ are contained in disjoint solid tori $V_{1}$ and $V_{2}$, respectively, the cores of which form a Hopf link. Note that $C$ is an untwisted double of the core of $V_{2}$. By Propositions 2.3 and 2.4 of [EKT03], $L=k \cup C$ is a prime link. 


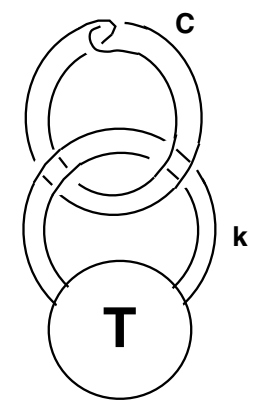

\section{Figure 2: The link $L$}

Let $K_{q}$ denote the knot obtained from $k$ by $1 / q$-surgery on the trivial knot $C$. We take $q>0$ for simplicity. It is helpful to view $K_{q}$ as a satellite of a $(-2 q)$-twist knot $\gamma_{2 q}$. (The notation $\gamma_{2 q}$ here means that the usual regular projection of $\gamma_{2 q}$ has $-2 q$ negative half-twists.) From this perspective, it follows immediately that each $K_{q}$ is nontrivial.

If $k$ is composite, then it follows from Theorem 4.1 of [G-S97] that $K_{q}$ is prime for $q \geq 3$, since $k \cup C$ is prime. On the other hand, if $k$ is prime and $K_{q^{\prime}}$ is composite for some $q^{\prime}$, then $K_{q}$ will be prime when $q \geq q^{\prime}+3$. Thus there exists $d \geq 3$ such that $K_{q}$ is prime whenever $q \geq d$; cf. Remarks 3.5 (ii).

For a fixed meridian-longitude pair $(m, l)$ of $k$, we have an epimorphism $\phi: \pi k \rightarrow G$ for which $\phi(m)=\mu$ and $\phi(l)=\lambda$. The elements $m$ and $l$ are represented by oriented simple closed curves $m_{1}$ and $l_{1}$ on the boundary of a tubular neighborhood of $k$ in $V_{1}$. Assume that $m_{1} \cap l_{1}$ is a single point $*$, which we take as the basepoint for $\pi(k \cup C), \pi K_{q}$ and $\pi k$, as previously mentioned. The curve $m_{1}$ certainly represents a meridian of $K_{q}$, and since the linking number of $k$ and $C$ is zero, the curve $l_{1}$ represents a longitude (see pages 266-267 of [R76]). Furthermore, if $(w, c)$ is a meridian-longitude pair for $C$ (based at $*$ ), we have $\pi k=\pi(k \cup C) /\langle\langle w\rangle\rangle$ and $\pi K_{q}=\pi(k \cup C) /\left\langle\left\langle w c^{q}\right\rangle\right\rangle$. (Here and throughout $\langle\langle\rangle\rangle$ denotes normal closure.) Thus

$$
\pi K_{q} /\langle\langle w\rangle\rangle=\pi(k \cup C) /\left\langle\left\langle w, w c^{q}\right\rangle\right\rangle=\pi k /\left\langle\left\langle c^{q}\right\rangle\right\rangle
$$

But $c=1$ in $\pi k$, since $C$ represents the identity element of $\pi k$, and so $\pi K_{q} /\langle\langle w\rangle\rangle=\pi k$. The induced homomorphism $\eta: \pi K_{q} \rightarrow \pi k$ is an epimorphism taking meridian to meridian and longitude to longitude, preserving orientations; cf. Remarks 3.5 (ii). Therefore, $\phi_{q}=$ $\phi \circ \eta: \pi K_{q} \rightarrow G$ is an epimorphism that maps the classes of $m_{1}$ and $l_{1}$ to $\mu$ and $\lambda$, respectively.

Recall that $K_{q}\left(q \geq d\right.$ from now on) is a satellite of a $(-2 q)$-twist knot $\gamma_{2 q}$. Since $\gamma_{2 q}$ is the double of the unknot with twisting number $-2 q$, it follows from the form of 
the Alexander polynomial for doubled knots (see p. 136 of [BZ85], for example) that the exteriors $\operatorname{Ext}\left(\gamma_{2 q}\right)$ and $\operatorname{Ext}\left(\gamma_{2 \bar{q}}\right)$ are not homeomorphic if $q \neq \bar{q}$.

Finally, consider the canonical splitting of the exterior $E_{q}$ of $K_{q}$ into a union $\Sigma_{q}$ of Seifert pieces (the characteristic submanifold of $E_{q}$ ) and a union $\Lambda_{q}$ of atoroidal pieces $\left(\Lambda_{q}=\operatorname{cl}\left(E_{q} \backslash \Sigma_{q}\right)\right)$ [JS79], [J76]. Each of $\Sigma_{q}$ and $\Lambda_{q}$ has a finite number of components, and

$$
\operatorname{cl}\left[\left(\Sigma_{q} \cup \Lambda_{q}\right) \backslash \operatorname{Ext}\left(\gamma_{2 q}\right)\right] \cong \operatorname{cl}\left[\left(\Sigma_{r} \cup \Lambda_{r}\right) \backslash \operatorname{Ext}\left(\gamma_{2 r}\right)\right],
$$

for $q, r \geq d$. Now choose $p \in\{d, d+1, \ldots\}$ so large that a copy of $\operatorname{Ext}\left(\gamma_{2 q}\right)$ is not a component of $\Lambda_{q} \backslash \operatorname{Ext}\left(\gamma_{2 q}\right)$, for any $q \geq p$. Thus if $q \neq r$ and $q, r \geq p$, then $E_{q}$ is not homeomorphic to $E_{r}, \operatorname{since} \operatorname{Ext}\left(\gamma_{2 q}\right)$ is not homeomorphic to $\operatorname{Ext}\left(\gamma_{2 r}\right)$. Therefore, there exist infinitely many distinct prime knots realizing $\lambda$.

Remarks 2.3. (i) Notice the seemingly large number of choices we have in the above construction of prime knots that realize $\lambda$. For example, we might double and redouble $C$ itself.

(ii) To see that the answer to each of Questions 1.1 and 1.2 is yes, see Example 2.6. Less specifically, let $G$ be the group of a composite knot $k$ with meridian-longitude pair $(\mu, \lambda)$, and let $k_{1}$ and $k_{2}$ be ambient isotopic copies of $k$. Assume that $k$ is oriented and that each of $k_{1}$ and $k_{2}$ inherits this orientation. Let $\left(m_{i}, l_{i}\right)$ be a meridian-longitude pair for $k_{i}(i=1,2)$, and let $\phi_{i}: \pi k_{i} \rightarrow \pi k$ be an isomorphism such that $\phi\left(m_{i}\right)=\mu$ and $\phi_{i}\left(l_{i}\right)=\lambda$. Then $\phi_{1}$ and $\phi_{2}$ induce an epimorphism $\phi: \pi\left(k_{1} \sharp k_{2}\right) \rightarrow G$ such that $\phi(m)=\mu$ and $\phi(l)=\lambda^{2}$, for some choice of meridian-longitude pair $(m, l)$ for $k_{1} \sharp k_{2}$. Thus $k_{1} \sharp k_{2}$ covers $\left(G, \mu, \lambda^{2}\right)$, and so there exist prime knots $K_{q}$ covering $\left(G, \mu, \lambda^{2}\right)$ by Theorem 2.2.

Corollary 2.4. Let $G$ be a knot group normally generated by $\mu \in G$, and let $\lambda \in G$. Then there exists an infinite number of prime knots realizing $\lambda$ if and only if $\lambda \in G^{\prime \prime} \cap Z(\mu)$, where $G^{\prime \prime}$ is the second commutator subgroup of $G$ and $Z(\mu)$ is the centralizer of $\mu$ in $G$.

Proof. According to the main result, Proposition 1, of [JL89], $\lambda$ is realizable (by some knot) if and only if $\lambda \in G^{\prime \prime} \cap Z(\mu)$, since $G$ is a knot group. But Theorem 2.2 implies that $\lambda$ is realizable if and only if it is realizable by an infinite number of prime knots.

Remarks 2.5. (i) If we take $\lambda$ to be the longitude of a knot group $G_{2}$ with meridian $\mu$, then Corollary 2.4 ensures the existence of a knot group $G_{1}$ not isomorphic to $G_{2}$ and an epimorphism $\phi: G_{1} \rightarrow G_{2}$ that preserves peripheral structure. This provides another answer to Questions 1.1 and 1.2.

(ii) One can avoid Corollary 2.4 by noting that the group of any knot $K$ covers itself by the identity automorphism $(\pi K, m, l) \rightarrow(\pi K, m, l)$, and then taking $k=K$ in the construction given in the proof of Theorem 2.2. 
Example 2.6. Consider the 2-component link $L=k \cup C$ in Figure 3. Regard the knotted component $k$ as the connected sum $\left(k_{W} \sharp k_{N}\right) \sharp\left(k_{E} \sharp k_{S}\right)$, of two Granny knots. (Here $W, N, E, S$ abbreviate west, north, east and south.) As in Remarks 2.3 (ii), there exist isomorphisms $\pi\left(k_{W} \sharp k_{N}\right) \rightarrow \pi\left(k_{W} \sharp k_{E}\right)$ and $\pi\left(k_{E} \sharp k_{S}\right) \rightarrow \pi\left(k_{W} \sharp k_{E}\right)$, taking the longitude of each of $\pi\left(k_{W} \sharp k_{N}\right)$ and $\pi\left(k_{E} \sharp k_{S}\right)$ to that of $\pi\left(k_{W} \sharp k_{E}\right)$. Hence there is an epimorphism $\phi: \pi k \rightarrow \pi\left(k_{W} \sharp k_{E}\right)$ that takes a longitude of $\pi k$ to the square of that of $\pi\left(k_{W} \sharp k_{E}\right)$. Moreover, $\phi$ maps the class of $C$ trivially.

Let $K_{q}$ be the knot resulting from $k$ after $1 / q$ surgery on $C$. As in the proof of Theorem 2.2, the group $\pi K_{q}$ admits an epimorphism onto the Granny knot group, sending meridian to meridan, longitude to the square of a longitude

We obtain the conclusion of Theorem 2.2 by another method, one that enables us to obtain hyperbolic knots $K_{q}$. Since the link $L$ is prime and alternating, a theorem of W. Menasco [Me84] implies that $L$ is hyperbolic. Results of W. Thurston [Th77/83] and W. Neumann and D. Zagier [NZ85] imply that for sufficiently large $q$ the knots $K_{q}$ are hyperbolic, with strictly increasing volumes (that approach $36.4732 \ldots$ ); in particular, the knots are distinct.

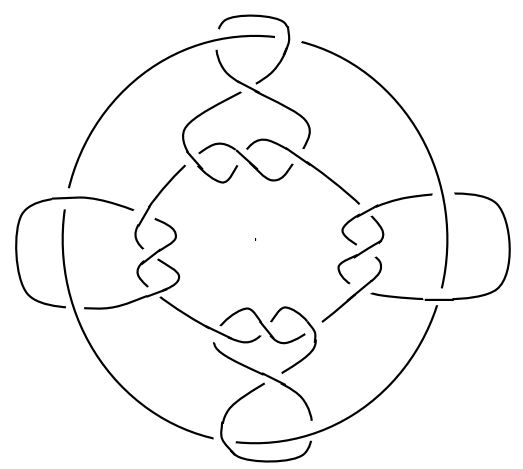

Figure 3: $k \cup C$

Remark 2.7. The choice of a curve $C$ as in Example 2.6 and in Theorem 2.2 satisfies four basic requirements:

1. $C$ is unknotted in $S^{3}$;

2. The linking number of $C$ and $k_{1} \sharp k_{2}$ is zero;

3. The link $C \cup\left(k_{1} \sharp k_{2}\right)$ is prime; and

4. The epimorphism $\phi: \pi\left(k_{1} \sharp k_{2}\right) \rightarrow \pi k$ maps the class of $C$ trivially.

3. Partial order on knots. Motivated by the results of $\S 2$, we introduce a relation on the set of all knots.

Definition 3.1. $k_{1} \succeq k_{2}$ if there is an epimorphism $\pi k_{1} \rightarrow \pi k_{2}$ preserving peripheral structure. 
Proposition 3.2. The relation $\succeq$ is a partial order.

Proof. Clearly $\succeq$ is reflexive and transitive. It remains to show that $\succeq$ is antisymmetric. If $k_{1} \succeq k_{2}$ and $k_{2} \succeq k_{1}$, then there exist epimorphisms $\phi: \pi k_{1} \rightarrow \pi k_{2}$ and $\eta: \pi k_{2} \rightarrow$ $\pi k_{1}$, preserving peripheral structure. The compositions $\phi \circ \eta$ and $\eta \circ \phi$ are epimorphisms from a knot group to itself. Since any knot group is residually finite [H87] and finitely generated, it has the Hopfian property: any epimorphism from the group to itself is an isomorphism [M40]. Hence both $\phi \circ \eta$ and $\eta \circ \phi$ are isomorphisms. In particular, it follows that $\phi$ is an isomorphism preserving peripheral structure. By [W68] $k_{1}$ and $k_{2}$ have homeomorphic complements. Finally [GL89] implies that $k_{1}$ and $k_{2}$ are of the same knot type (not necessarily of the same ambient isotopy type, however, since chiral knots exist)

Remarks 3.3. (i) The condition that the epimorphism preserve peripheral structure is needed for the conclusion of Proposition 3.2. To see that this is so, let $k_{1}$ be the granny knot and $k_{2}$ the square knot. Since their groups are isomorphic, we certainly have epimorphisms from one to the other. However, no such epimorphism preserves peripheral structure, and indeed $k_{1}$ is not equal to $k_{2}$.

(ii) The relation $\succeq$ is compatible with some well-known invariants. For example, if $\Delta_{k}^{(i)}(t)(i \geq 1)$ denotes the $i$ th Alexander polynomial of $k$, then $k_{1} \succeq k_{2}$ implies the existence of an epimorphism $\pi k_{1} \rightarrow \pi k_{2}$, which in turn implies that, for each $i, \Delta_{k_{1}}^{(i)}(t)$ contains $\Delta_{k_{2}}^{(i)}(t)$ as a factor. Following the usual practice, we will abbreviate $\Delta_{k}^{(1)}(t)$ by $\Delta_{k}(t)$ and refer to it as the Alexander polynomial of $k$. Necessary algebraic background information can be found in [MKS76]).

(iii) If $k=k_{1} \sharp k_{2}$, then $k \succeq k_{1}$ and $k \succeq k_{2}$. Hence $\succeq$ refines the crude partial order induced by knot factorization.

There are several natural methods to produce knots $k_{1}, k_{2}$ with $k_{1} \succeq k_{2}$. For example, suppose that a diagram for a knot $k_{1}$ displays a rotational symmetry $f$. Let $k_{2}$ denote the quotient knot in the 3 -sphere $S^{3} / f$. By identifying all pairs of Wirtinger generators corresponding to arcs in $f$-orbits we obtain a projection from $\pi k_{1}$ to $\pi k_{2}$ preserving peripheral structures. Hence $k \succeq \bar{k}$.

Another method is contained in the following.

Proposition 3.4. Assume that $k$ is a satellite knot with pattern $\operatorname{knot} \tilde{k}$. Then $k \succeq \tilde{k}$.

Proof. The satellite knot $k$ is the image of a diffeomorphism $g: \tilde{V} \rightarrow \hat{V} \subset S^{3}$, where $\tilde{V}$ is a standard solid torus containing $\tilde{k}$, and $\hat{V}$ is a tubular neighborhood of a knot $\hat{k}$, the companion knot. As usual, we require that $\tilde{k}$ not be contained in any 3 -ball of $\tilde{V}$, and 
also that $g$ send the longitude $l$ of $\tilde{V}$ to the longitude $\hat{l}$ of the solid torus $\hat{V}$. Denote the meridian of $\hat{V}$ by $\hat{m}$.

The group $\pi k$ of the satellite is isomorphic to the free product of $\pi \hat{k}$ and $\pi_{1}(\tilde{V} \backslash \tilde{k})$ with amalgamation: subgroup $\langle\hat{m}, \hat{l}\rangle$ of $\pi \hat{k}$ is identified with the subgroup $\langle m, l\rangle$ of $\pi_{1}(\tilde{V} \backslash \tilde{k})$, matching $\hat{m}$ and $\hat{l}$ with $m$ and $l$ (see [BZ85], for example). By a well-known property of free products with amalgamation, both $\pi \hat{k}$ and $\pi_{1}(\tilde{V} \backslash \tilde{k})$ are subgroups of $\pi k$ embedded in the obvious way. (see [LS77]).

Consider the natural projection $\phi: \pi k \rightarrow \pi k / N$ where $N$ is the normal closure of the commutator subgroup of $\pi \hat{k}$. Regard $\pi k / N$ as the quotient group of $\pi k$ obtained by allowing the elements of $\pi \hat{k}$ to commute. In the quotient, $\pi \hat{k}$ collapses to the infinite cyclic group generated by $\hat{m}$. The effect on $\pi_{1}(\tilde{V} \backslash \tilde{k})$ is to kill $l$, resulting in the group $\pi \tilde{k}$. Hence $\phi$ is an epimorphism from $\pi k$ to $\pi \tilde{k}$. It is obvious that $\phi$ preserves peripheral structure.

Remarks 3.5. (i) Proposition 3.4 states that a satellite knot $k$ covers its pattern knot $\tilde{k}$. It is not generally true that $k$ covers its companion $\hat{k}$. To see this, consider the untwisted double $k$ of the trefoil knot. The Alexander polynomial $\Delta_{k}(t)$ is trivial (see [BZ85]). However, the companion knot is the trefoil, which has nontrivial Alexander polynomial. In view of Remark 3.3 (ii), $k$ does not cover $\hat{k}$. We mention that for a given satellite knot, the pattern knot might well cover the companion or vice versa.

(ii) We can avoid the use of Theorem 4.1 of [G-S97] and apply Proposition 3.4 to give an alternative proof of Theorem 2.2 as follows. Think of $K_{q}$ as the satellite knot with pattern $k$ in the interior of the (standardly embedded) solid torus $V_{1}$ and with companion the twist-knot $\gamma_{2 q}$; here $V_{1}$ is mapped by a longitude-preserving homeomorphism onto a tubular neighborhood of $\gamma_{2 q}$. Since $\operatorname{Int}\left(V_{1}\right)$ contains no 2-sphere that decomposes $K_{q}$ as a nontrivial connected sum, it follows easily that $K_{q}$ is prime for $q \geq 2$, say. Proposition 3.4 immediately yields the epimorphism $\eta: \pi K_{q} \rightarrow \pi k$. We omit details.

Recall that the genus $g(k)$ of a knot $k$ is the smallest genus of any Seifert surface of $k$.

Conjecture 3.6. If $k_{1} \succeq k_{2}$ then $g\left(k_{1}\right) \succeq g\left(k_{2}\right)$.

As evidence for Conjecture 3.6 we offer the following.

Proposition 3.7. Assume that $k_{1} \succeq k_{2}$. If either (i) $k_{1}$ is fibered or (ii) $k_{2}$ is alternating then $g\left(k_{1}\right) \succeq g\left(k_{2}\right)$.

Proof. (i) A knot $k$ is fibered if and only if the commutator subgroup $[\pi k, \pi k]$ is finitely generated, in which case it is a free group of rank equal to $2 g(k)$ (see [R76], for example). Assume that $k_{1} \succeq k_{2}$. The epimorphism $\pi k_{1} \rightarrow \pi k_{2}$ restricts to an epimorphism of 
commutator subgroups. Consequently, $\left[\pi k_{2}, \pi k_{2}\right]$ is finitely generated, and hence free of rank less than or equal that of $\left[\pi k_{1}, \pi k_{1}\right]$. Since the rank of $\left[\pi k_{2}, \pi k_{2}\right]$ is equal to $2 g\left(k_{2}\right)$, the proof is complete.

(ii) By a theorem of Seifert [S34], for any knot $k$ we have $2 g(k) \geq \operatorname{deg} \Delta_{k}(t)$, while a theorem of Murasugi [M60] states that equality holds when $k$ is alternating. Assume that $k_{1} \succeq k_{2}$. Then $\Delta_{k_{2}}(t)$ divides $\Delta_{k_{1}}(t)$. Hence $2 g\left(k_{1}\right) \geq \operatorname{deg} \Delta_{k_{1}}(t) \geq \operatorname{deg} \Delta_{k_{2}}(t)=2 g\left(k_{2}\right)$.

There is further evidence for Conjecture 3.6. If $k_{1} \succeq k_{2}$, then the epimorphism $\pi\left(k_{1}\right) \rightarrow \pi\left(k_{2}\right)$ is induced by a boundary-preserving map $f: \operatorname{Ext}\left(k_{1}\right) \rightarrow \operatorname{Ext}\left(k_{2}\right)$. If $S$ is a minimal genus Seifert surface spanning $k_{1}$, and if $f_{*}([S]) \in H_{2}\left(\operatorname{Ext}\left(k_{2}\right), \partial \operatorname{Ext}\left(k_{2}\right)\right)(\cong \mathbb{Z})$ is not zero, then it follows from Corollary 6.22 of [G83] that $g\left(k_{1}\right) \geq g\left(k_{2}\right)$. We are grateful to Ian Agol for pointing out the connection between Conjecture 3.6 and Gabai's result.

Proposition 3.8. If $k_{i}$ is a sequence of hyperbolic knots such that $k_{0} \succeq k_{1} \succeq \cdots \succeq k_{i} \succeq$ $\cdots$, then $k_{i}=k_{i+1}$ for sufficiently large $i$.

Proof. Assume that $k_{0} \succeq k_{1} \succeq k_{2} \succeq \cdots$, for hyperbolic knots $k_{i}$. Consequently, there is a sequence of epimorphisms

$$
\pi k_{0} \stackrel{\phi_{0}}{\longrightarrow} \pi k_{1} \stackrel{\phi_{1}}{\longrightarrow} \pi k_{2} \stackrel{\phi_{2}}{\longrightarrow} \cdots
$$

each preserving peripheral structure. By Theorem 1 of [S02], $\phi_{i}$ is an isomorphism for sufficiently large $i$. (The result of [S02] requires only knot group epimorphisms without any constraint on peripheral subgroups.) As in the proof of Proposition 3.2, [W68] and [GL89] together imply that $k_{i}=k_{i+1}$ for sufficiently large $i$

Conjecture 3.9. (Cf. J. Simon: Problem 1.12 (D) in [K95]) ] Any knot covers only finitely many knots. In other words, if $k_{1}$ is a knot, then the collection of all knots $k_{2}$ such that $k_{1} \succeq k_{2}$ is finite.

Conjecture 3.9 is true if $k_{1}$ is fibered. To see this, note that $k_{2}$ must also be fibered. In [S95] an entropy invariant $h_{k}$ was defined for any fibered knot $k$. If $k_{1} \succeq k_{2}$, then $h_{k_{1}} \geq h_{k_{2}}$ and also $g\left(k_{1}\right) \geq g\left(k_{2}\right)$. By Theorem 3.4 of [S95], there exist only finitely many fibered knots with genus and entropy no greater than given bounds.

Definition 3.10. A knot $k$ is minimal (with respect to the partial order) if $k \succeq k^{\prime}$ implies that $k=k^{\prime}$ or else $k^{\prime}$ is trivial.

Proposition 3.11. If $k$ is a fibered knot with irreducible Alexander polynomial, then $k$ is minimal. 
Proof. Assume that $k \succeq k^{\prime}$. Since $\Delta_{k^{\prime}}(t)$ divides $\Delta_{k}(t)$ and $\Delta_{k}(t)$ is irreducible, either $\Delta_{k^{\prime}}(t)=\Delta_{k}(t)$ or else $\Delta_{k^{\prime}}(t)=1$. In the first case, the epimorphism $\phi: \pi k \rightarrow \pi k^{\prime}$ restricts to an isomorphism of commutator subgroups, as these groups are both free of rank equal to $\operatorname{deg} \Delta_{k}(t)$; it follows that $\phi$ is itself an isomorphism, and as in the proof of Proposition $3.2, k=k^{\prime}$. In the second case, $\Delta_{k^{\prime}}(t)=1$, and this together with the fact that $k^{\prime}$ is fibered imply that $k^{\prime}$ is trivial.

The figure eight knot $4_{1}$ is minimal by Proposition 3.11. However, Example 3.12 below shows that $4_{1}$ does not remain minimal in the larger category of virtual knots. It shows also that Conjecture 3.9 is not true in the category.

A knot is often studied as an equivalence class of planar knot diagrams, two diagrams being equivalent if one can be obtained from the other by a sequence of Reidemeister moves. In 1997 Kauffman introduced virtual knot diagrams, allowing a new type of crossing, called a virtual crossing and indicated by a small circle surrounding the site. After suitably extending the usual Reidemeister moves to allow certain deformations involving virtual crossings, Kauffman defined a virtual knot to be an equivalence class of virtual diagrams. The reader is referred to [K97], [K99], [K00] for details. It is a remarkable feature of the theory that two classical knots are equivalent under generalized Reidemeister moves if and only if they are equivalent under the classical ones [GPV00]. In this sense, virtual knot theory is an extension of the classical theory.

Many classical invariants of knot theory extend naturally in the larger virtual category. In particular, one can associate a knot group to an equivalence class of diagrams. Virtual knot groups were classified in [SW00] both in terms of combinatorial presentations and topologically (see also [Ki00]). The peripheral structure of a virtual knot is defined just as for classical knots (see [Ki00] for details).

Example 3.12. A diagram for the figure eight knot appears in Figure 4 with labeled Wirtinger generators. The group of $k$ has a presentation $\langle x, y, z, w| z x=y z, y w=$ $z y, w x=z w\rangle$. (The fourth Wirtinger relation $x w=y x$ is a consequence of the other three, and so we have omitted it.) Using the second and third relations to express $w$ and $z$ in terms of $x$ and $y$, we see that

$$
\pi k \cong\left\langle x, y \mid x^{-1} y^{-1} x y^{-1} x^{-1} y x y^{-1} x y\right\rangle .
$$

Consider the diagram for the virtual knot $k_{q}(q \geq 2)$ in Figure 4 with labeled Wirtinger generators. The corresponding group generated by $x, y, z, w, w_{1}, \ldots, w_{q-1}$ with relations $z x=y z, y w=z y, w x=z w, x w_{1}=w x, x w_{2}=w_{1} x, \ldots, x w_{q-2}=w_{q-3} x, w y=w_{q-2} x$. 

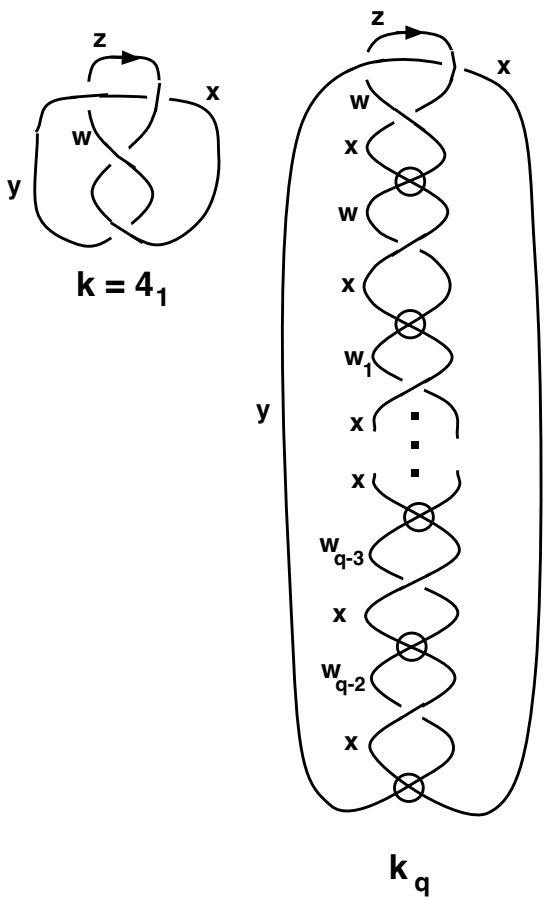

$(q>1)$

\section{Figure 4: Figure eight knot and $K_{q}$}

The fourth relation implies that $w_{1}=x^{-1} w x$. The fifth implies that $w_{2}=x^{-2} w x^{2}$, and so forth. The next to last relation implies that $w_{q-2}=x^{-(q-2)} w w^{q-2}$. We use these to eliminate $w_{1}, \ldots, w_{q-2}$. The last relation then becomes $x^{-q} y x^{q}=y$. Consequently,

$$
\pi k_{q} \cong\left\langle x, y, z, w \mid z x=y z, y w=z y, w x=z w, y=x^{-(q-1)} y x^{q-1}=w\right\rangle .
$$

Recall that the three relations $z x=y z, y w=z y, w x=z w$ together imply that $x w=y x$, which can be rewritten as $w=x^{-1} y x$. Substitution in the relation $y=x^{-(q-1)} y x^{q-1}=w$ yields

$$
\begin{gathered}
\pi k_{q} \cong\left\langle x, y, z, w \mid z x=y z, y w=z y, w x=z w, x^{-q} y x^{q}=y\right\rangle \\
\cong \pi k /\left\langle\left\langle x^{-q} y x^{q}=y\right\rangle\right\rangle \\
\cong\left\langle x, y \mid x^{-1} y^{-1} x y^{-1} x^{-1} y x y^{-1} x y, x^{-q} y x^{q}=y\right\rangle
\end{gathered}
$$

The Reidemeister-Schreier method (see [LS77], for example) can be used to find a presentation for the commutator subgroup of $\pi k_{q}$ :

$$
\left\langle a_{j} \mid a_{j+2}=a_{j+1}^{2} a_{j}^{-1} a_{j+1}, a_{j+q}=a_{j} \quad(j \in \mathbf{Z})\right\rangle .
$$


(This group is in fact the fundamental group of the $q$-fold cyclic cover of $S^{3}$ branched over $k$. However, we do not require this fact.) Its abelianization is finite, and it has order equal to the absolute value of the cyclic resultant $\operatorname{Res}\left(t^{2}-3 t+1, t^{q}-1\right)$. These values grow exponentially with $q$ (see [SW02]). Consequently, the groups $\pi k_{q}$ are pairwise nonisomorphic for sufficiently large $q$. (In fact, they are all nonisomorphic, but this is again a fact that we do not require.)

The canonical projection $\phi_{q}: \pi k \rightarrow \pi k_{q}$ is a surjection that maps the elements $x$ and $z^{-1} x y^{-1} w$, forming a peripheral pair for $k$, to their cosets in $\pi k_{q}$. It is easy to see that $\left(x, z^{-1} x^{-(q-1)} y^{-1} w x^{n-2}\right)$ is a peripheral pair for $k_{q}$. Using the relations above, one checks that $z^{-1} x^{-(q-1)} y^{-1} w x^{n-2}$ is equal to $z^{-1} x y^{-1} w$. Hence the projection $\phi_{q}$ preserves peripheral structure, and we have produced infinitely many virtual knots $k_{q}$ such that $k \succeq k_{q}$.

4. Degree one maps and a related partial order. The partial order in Definition 3.1 is related to another, a partial order on compact 3-manifolds, one that has been studied by Y. Rong [R92], S. Wang [W02], and others: If $M$ and $N$ are compact, oriented 3-manifolds, then $M \succeq_{1} N$ if there exists a degree one proper map from $M$ to $N$. (A manifold map is proper if it maps boundary to boundary.) When applied to exteriors of knots $k_{1}, k_{2}$, the relation becomes: $k_{1} \succeq_{1} k_{2}$ if there is an epimorphism $\pi k_{1} \rightarrow \pi k_{2}$ mapping meridian $m_{1}$ to $m_{2} l_{2}^{p}$ and longitude $l_{1}$ to $m_{2}^{q} l_{2}^{ \pm 1}$, for some integers $p, q$. Since the normal subgroup generated by $m_{2} l_{2}^{p}$ must be all of $\pi k_{2}$, Corollary 2 of [CGLS87] implies that $p \in\{0,1,-1\}$. (The recent proof that every nontrivial knot satisfies Property $\mathrm{P}$ [KM04] implies that $p$ must in fact be 0 . However, we do not require that fact.) Also, since $m_{2}^{q} l_{2}^{ \pm 1}$ must be in $\left(\pi k_{2}\right)^{\prime \prime} \cap Z\left(m_{2}\right)$ [JL89], we have $q=0$.

Clearly, $k_{1} \succeq_{1} k_{2}$ implies $k_{1} \succeq k_{2}$.

Theorem 4.1. In general, the relation $k_{1} \succeq k_{2}$ does not imply $k_{1} \succeq_{1} k_{2}$.

Proof. Let $k_{1}, k_{2}$ be the torus knots $9_{1}, 3_{1}$, respectively. Since $k_{1}$ has a rotational symmetry with $k_{2}$ as quotient, it follows from the remark before Proposition 3.4 that $k_{1} \succeq k_{2}$.

Note that the epimorphism $\pi k_{1} \rightarrow \pi k_{2}$ induced by the symmetry maps meridian $m_{1}$ to meridian $m_{2}$ and longitude $l_{1}$ to the third power $l_{2}^{3}$. It suffices to show there exists no epimorphism mapping $\left(m_{1}, l_{1}\right)$ to $\left(m_{2} l_{2}^{p}, l_{2}^{ \pm 1}\right)$, for $p \in\{0,1,-1\}$. Since torus knots satisfy property $\mathrm{P}$ [S33] (see also [H64] or [BZ85], p.274), the integer $p$ must be zero. The desired conclusion follows from the next proposition. (Details are given in Example 4.3.)

The A-polynomial was introduced in [CCGLS94]. We briefly review its definition, following [CL96]. For any knot $k$ with meridian-longitude pair $(m, l)$, consider the affine algebraic set $R=\operatorname{Hom}\left(\pi k, S L_{2}(\mathbf{C})\right)$. Let $R_{\triangle}$ be the algebraic subset consisting of all 
$\rho \in R$ such that $\rho(l)$ and $\rho(m)$ are upper triangular. Let $\xi: R_{\triangle} \rightarrow \mathbf{C}^{2}$ be the eigenvalue map $\rho \mapsto(M, L)$, where $M$ and $L$ are the top-left entries (eigenvalues) of $\rho(m)$ and $\rho(l)$, respectively. The closure of any component of $\xi\left(R_{\triangle}\right)$ has complex dimension 0 or 1. Each 1-dimensional component is the zero set of a polynomial that is unique up to multiplication by a constant; the product of all such polynomials, divided by $L-1$ (the polynomial corresponding to abelian representations) is $A_{k}$. It is possible to normalize, choosing a suitable multiplicative constant, so that the coefficients of $A_{k}$ are integers with no common divisor, and we do so.

Proposition 4.2. Let $k_{1}, k_{2}$ be knots. If $\phi: \pi k_{1} \rightarrow \pi k_{2}$ is a homomorphism mapping $\left(m_{1}, l_{1}\right)$ to $\left(m_{2}^{a} l_{2}^{b}, m_{2}^{c} l_{2}^{d}\right)$, for integers $a, b, c, d$, then each irreducible factor of $A_{k_{2}}(M, L)$ divides $\left(M^{c} L^{d}-1\right) \cdot A_{k_{1}}\left(M^{a} L^{b}, M^{c} L^{d}\right)$.

Proof. Denote the eigenvalue maps for $k_{1}$ and $k_{2}$ by $\xi_{1}$ and $\xi_{2}$, respectively. If $(M, L)$ is in the image of $\xi_{2}$, then $\left(M^{a} L^{b}, M^{c} L^{d}\right)$ is in the image of $\xi_{1}$. Hence $A_{k_{2}}(M, L)=0$ implies that $\left(M^{c} L^{d}-1\right) \cdot A_{k_{1}}\left(M^{a} L^{b}, M^{c} L^{d}\right)=0$. The result follows from Hilbert's Nullstellensatz (see [H77], for example).

Example 4.3. The A-polynomial of $k_{1}=9_{1}$ is $1+M^{18} L$ while that of the trefoil $k_{2}=3_{1}$ is $1+M^{6} L$ (see [CCGLS94] or [N02]). The polynomial $A_{3_{1}}(M, L)$ divides $A_{9_{1}}\left(M, L^{3}\right)$, reflecting the fact that there is a homomorphism $\pi k_{1} \rightarrow \pi k_{2}$ mapping $\left(m_{1}, l_{1}\right)$ to $\left(m_{2}, l_{2}^{3}\right)$. However, the polynomial $A_{3_{1}}(M, L)$ is irreducible and divides neither $A_{9_{1}}(M, L)$ nor $A_{9_{1}}\left(M, L^{-1}\right)$. Hence by Proposition 4.2 , there is no homomorphism $\pi k_{1} \rightarrow \pi k_{2}$ mapping $\left(m_{1}, l_{1}\right)$ to $\left(m_{2}, l_{2}\right)$ or $\left(m_{2}, l_{2}^{-1}\right)$.

Acknowledgement. The second author wishes to thank the Department of Mathematics of the University of Virginia for its generous hospitality and the use of its facilities.

\section{References}

[BZ85] G. Burde and H. Zieschang, Knots, Walter de Gruyter, Berlin, 1985.

[CCGLS94] D. Cooper, M. Culler, H. Gillet, D.D. Long and P.B. Shalen, Plane curves associated to character varieties of knot complements, Invent. Math. 118 (1994), 47-84.

[CL96] D. Cooper and D.D. Long, Remarks on the A-polynomial of a knot, J. Knot Theory and its Ramif. 5 (1996), 609-628.

[CL98] D. Cooper and D.D. Long, Representation theory and the A-polynomial of a knot, Chaos, Solitons \& Fractals 9 (1998), 749-763.

[CGLS87] M. Culler, C. McA. Gordon, J. Luecke and P.B. Shalen, Dehn surgery on knots, Ann. Math. 125 (1987), 237-300. 
[EKT03] S. Eliahou, L. Kauffman and M. Thistlethwaite, Infinite families of links with trivial Jones polynomial, Topology 42 (2003), 155-169.

[G83] Gabai, David, Foliations and the topology of 3-manifolds, J. Diff. Geometry 18 (1983), 445-503.

[G-S97] C. Goodman-Strauss, On composite twisted unknots, Trans. Amer. Math. Soc. 349 (1997), 4429-4463.

[GL89] C.McA. Gordon and J. Luecke, Knots are determined by their complements, J. Amer. Math. Soc. 2 (1989), 371-415.

[H77] R. Hartshorne, Algebraic geometry, Springer, Berlin, 1977.

[H64] J. Hempel, A simply-connected 3-manifold is $S^{3}$ if it is the sum of a solid torus and the complement of a torus knot, Proc. Amer. Math. Soc. 15 (1964), 1954-1958.

[H87] J. Hempel, Residual finiteness for 3-manifolds, in Comb. Group Theory and Topology, Annals of Math. Studies 111 (1987), 379-396.

[JS79] W.H. Jaco and P.B. Shalen, Seifert fibered spaces in 3-manifolds, Mem. Amer. Math. Soc. 21 (1979), no. 220.

[J79] K. Johannson, Homotopy equivalence of 3-manifolds with boundaries, LNM 761, Springer-Verlag, Berlin, 1979.

[JL89] D. Johnson and C. Livingston, Periperally specified homomorphisms of knot groups, Trans. Amer. Math. Soc. 311 (1989), 135-146.

[K97] L.H. Kauffman, Talks at MSRI meeting in January 1997, AMS meeting at University of Maryland, College Park in March 1997, Isaac Newton Institute Lecture in November 1997, Knots in Hellas Meeting in Delphi, Greece in July 1998, APCTP-NANKAI Symposium on Yang-Baxter Systems, Non-Linear Models and Applications at Seoul, Korea in October 1998.

[K99] L.H. Kauffman, Virtual knot theory, European J. Comb. 20 (1999) 663-690.

[K00] L.H. Kauffman, A survey of virtual knot theory, in Knots in Hellas '98, ed. by C. McA. Gordon, V.F.R. Jones, L.H. Kauffman, S. Lambropoulou, J.H. Przytycki, World Scientific, Singapore 2000, 143-202.

[Ki00] S.-K. Kim, Virtual knot groups and peripheral structure, J. Knot Theory Ramif. 9 (2000), 797-812

[K95] R. Kirby, Problems in Low-Dimensional Topology, preprint (1995), http://www.math.berkeley.edu kirby/.

[KM04] P.B. Kronheimer and T.S. Mrowka, Witten's conjecture and Property P, preprint, 2004 . 
[LS77] R.C. Lyndon and P.E. Schupp, Combinatorial Group Theory, Springer-Verlag, Berlin, 1977.

[MKS76] W. Magnus, A. Karrass and D. Solitar, Combinatorial Group Theory, Second Edition, Dover, NY, 1976.

[M40] A. I. Mal'cev, On isomorphic matrix representations of infinite groups, Math. Sb. 8 (1940), 405-421.

[M60] K. Murasugi, On alternating knots, Osaka Math. J. 12 (1960), 277-303.

[N02] F. Nagasato, An approach to the A-polynomial of $(2,, 2 p+1)$-torus knots from Frohman-Gelca-Lofaro theory, preprint, 2002 http://harold.math.kyushu-u.ac.jp/

[NZ85] W.D. Neumann and D. Zagier, Volumes of hyperbolic three-manifolds, Topology 24 (1985), 307-322.

[R76] D. Rolfsen, Knots and Links, Publish or Perish, Inc., Berkeley, CA 1976.

[S33] H. Seifert, Topologie dreidimensionaler gefaserter Räume, Acta Math. 60 (1933), $147-238$.

[S34] H. Seifert, Über das Geschlecht von Knoten, Math. Annalen 110 (1934), 571-592.

[S95] D.S. Silver, Knot invariants from topological entropy, Top. Appl. 61 (1995), 159-177.

[SW00] D.S. Silver and S.G. Williams, Virtual knot groups, in Knots in Hellas '98, Proceedings of the International Conference on Knot Theory and its Ramifications (C. McA. Gordon, V.F.R. Jones, L.H. Kauffman, L. Lambropoulou and J.H. Przytycki, eds) World Scientific, Singapore, 2000, 440-451.

[SW02] D.S. Silver and S.G. Williams, Torsion numbers of augmented groups, L'Enseign. Math. 48 (2002), 317-343.

[S02] T. Soma, Epimorphism sequences between hyperbolic 3-manifold groups, Proc. Amer. Math. Soc. 130 (2002), 1221-1223.

[T77/83] W. Thurston, The geometry and topology of 3-manifolds (mimeographed notes), Princeton Univ., 1977/78.

[W68] F. Waldhausen, On irreducible 3-manifolds which are sufficiently large, Annals Math. 87 (1968), 56-88.

[W02] S. Wang, Non-zero degree maps between 3-manifolds, Proceedings of ICM, Volume II, Higher Ed. Press, Beijing, 2002, 457-468.

Department of Mathematics and Statistics, University of South Alabama Mobile, AL 36688 USA; silver@jaguar1.usouthal.edu

bjwcw@aol.com 